\title{
CANCER IMMUNOTHERAPY
}

\section{Smarter bombs for cancer treatment}

Tang, L. et al. Nat. Biotechnol. https://doi.org/10.1038/nbt.4181 (2018)

One of the more promising forms of immunotherapy for cancer is adoptive cell transfer (ACT), where $\mathrm{T}$ cells removed from the patient are proliferated and returned to fight the malignancy. While ACT has proven effective against liquid cancers and some solid tumors, it is still early days for the technology. This treatment modality is a focus of Darrell Irvine, a professor in the Department of Biological Engineering at the Massachusetts Institute of Technology. Irvine describes how with ACT a primary challenge is keeping $\mathrm{T}$ cells functional.

"We know of a variety of drugs that could be administered to promote $\mathrm{T}$ cell function, but a problem with many of these approaches is that if you systemically infuse these agents that promote $\mathrm{T}$ cell function they'll also often be accompanied by a lot of toxicity-because you're basically stimulating $\mathrm{T}$ cells everywhere in the body", he adds.

In previous work, the Irvine group had developed a method to "backpack" cytokines, which enhance the immune response by autocrine activation, in liposomes attached to T cells; however, adjuvant loading capacity and release were not optimal. In a new report in Nature Biotechnology, the lab modified their backpack in two significant ways. First, investigators formulated a cytokine, interleukin-15 super-agonist (IL15Sa), as a protein nanogel via a redox sensitive disulfide crosslinker. This approach increased the amount of the drug loaded on T cells by 100 -fold over the earlier method. And because activated T cells have a reducing cell surface relative to naïve T cells, disulfide cleavage allowed a steady release of the drug at the tumor and tumor draining lymph nodes. Second, after seeking out a stable cell surface protein to tether their cargo to, authors added CD45 antibodies to the nanogel that kept the particles at the cell surface, avoiding endocytosis and thereby prolonging cellular activation.

In experimental comparisons of their modified backpack to a basic injected $\mathrm{T}$ cell treatment or T cells plus infused IL15Sa, the backpack strategy delivered significantly improved outcomes in a syngeneic murine system for melanoma. Furthermore, when this strategy was tested with chimeric antigen receptor (CAR)- $\mathrm{T}$ cell therapy-a form of ACT where T cells are engineered to recognize tumor-specific antigens-with a xenogeneic glioblastoma model, backpack/CAR-T treatment eliminated tumors in four of five mice. Alternatively, CAR-T treatment alone or in combination with infused IL15SA yielded no difference or modest improvement.

With the technology licensed to Torque Therapeutics, clinical trials begin next year. In terms of further development, Irvine added, "We're still exploring other kinds of payloads the $\mathrm{T}$ cells could be armed with to make them function better or to act on other cells of the tumor microenvironment."

\section{Clark Nelson}

Published online: 24 August 2018 https://doi.org/10.1038/s41684-018-0137-1

\section{What Control Diet are you using?}
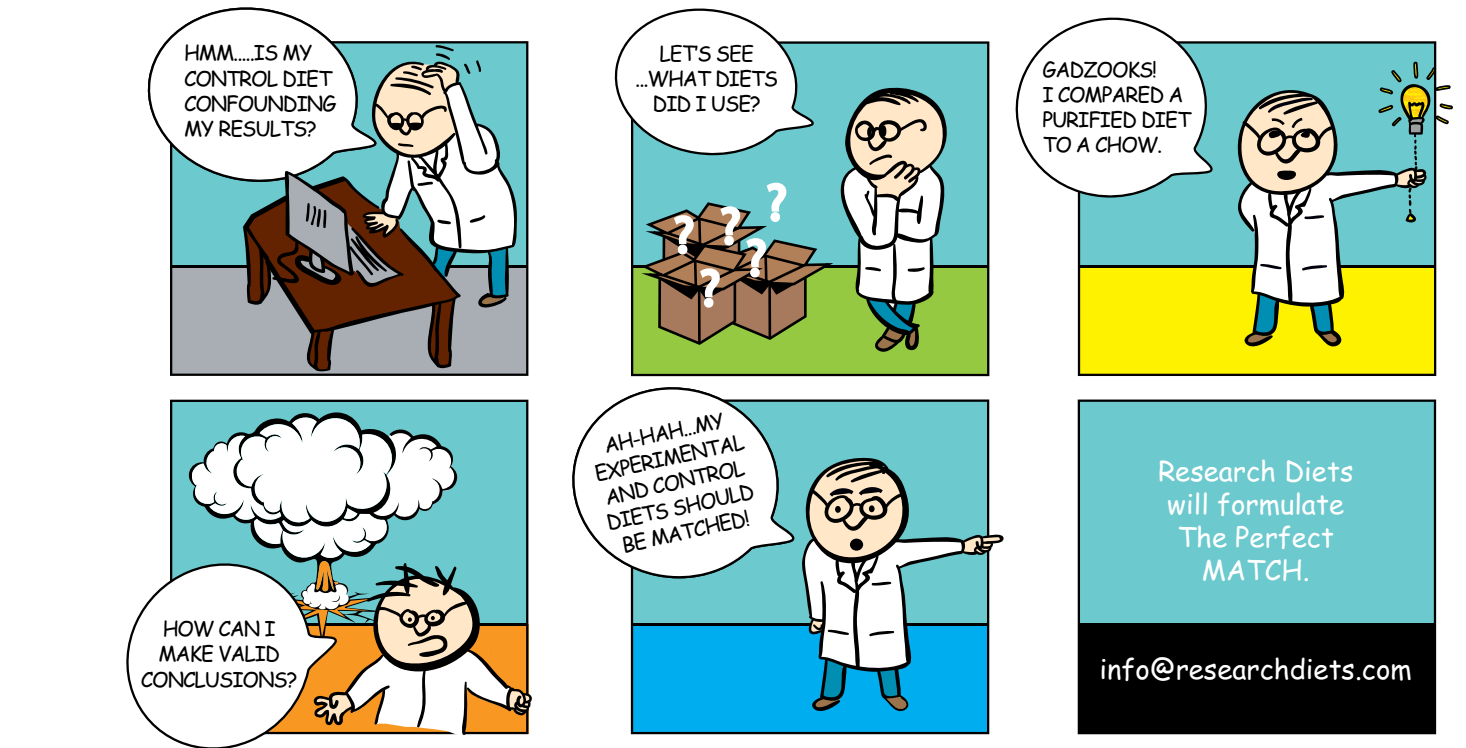

"We control the heck out of our diets."
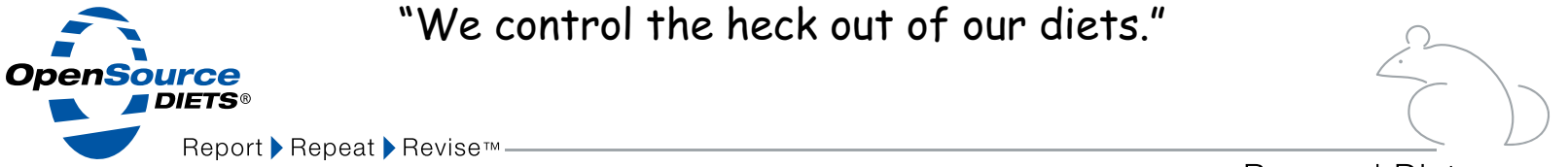

ResearchDiets.com 